\title{
Science with EPICS, the E-ELT planet finder
}

\author{
Raffaele Gratton $^{1}$, Markus Kasper ${ }^{2}$, Christophe Vérinaud ${ }^{3}$, \\ Mariangela Bonavita ${ }^{1,4}$ and Hans M. Schmid ${ }^{5}$ \\ ${ }^{1}$ INAF-Osservatorio Astronomico di Padova, Italy \\ email: raffaele.gratton@oapd.inaf.it \\ ${ }^{2}$ European Southern Observatory, Germany \\ ${ }^{3}$ Laboratoire de Astrophysique de Grenoble, France \\ ${ }^{4}$ University of Toronto, Canada \\ ${ }^{5}$ ETH Zurich, Switzerland
}

\begin{abstract}
EPICS is the proposed planet finder for the European Extremely Large Telescope. EPICS is a high contrast imager based on a high performing extreme adaptive optics system, a diffraction suppression module, and two scientific instruments: an Integral Field Spectrograph (IFS) for the near infrared (0.95-1.65 $\mu \mathrm{m})$, and a differential polarization imager (E-POL). Both these instruments should allow imaging and characterization of planets shining in reflected light, possibly down to Earth-size. A few high interesting science cases are presented.
\end{abstract}

Keywords. planetary systems, instrumentation: adaptive optics

\section{EPICS at E-ELT}

Direct observations of exosolar planets are among the drivers for the construction of E-ELT, the ESO project for an extremely large telescope (diameter of $42 \mathrm{~m}$ ). These observations require very high contrasts at very tiny separations from relatively bright sources, and are limited by background and diffraction. From very generic arguments, contrast in this regime is expected to depend on the square of telescope diameter at separations inversely proportional to this parameter. Although actually reaching such performances will be technically very challenging and possibly such a full gain will not be achieved, the gain of E-ELT with respect to existing or designed instrument is expected to be enormous.

EPICS is the instrument proposed for direct imaging and characterization (both in spectroscopy and polarimetry) of exoplanets. To explore its science potential, we notice that GPI and SPHERE are expected to achieve a photon noise limited contrast performance of better than $10^{-7}$ at 0.5 separation (Marois et al. 2008, Beuzit et al. 2008) using 8-m class telescopes. Hence a similar instrument at a 42-m telescope should be able to reach a 28 times better contrast at a 5 times smaller angular separation. EPICS should then reach $\sim 10^{-9}$ contrast at 0.1 separation.

Similar to SPHERE, EPICS baseline concept will include an extreme adaptive optics module (XAO), able to provide high Strehl. Due to lack of photons (a XAO system should sample the pupil with a step of $\sim 20 \mathrm{~cm}$ ), the reference source should be bright $(I<9)$. A second module will allow efficient suppression of the central diffraction peak and observations down to an inner working angle (IWA) of a few $\lambda / \mathrm{D}(0.03$ arcsec, with a goal of 0.02 arcsec). A set of scientific instruments will exploit simultaneous differential imaging techniques. The Integral Field Spectrograph (IFS) will image at Nyquist limit a square field of view (FoV) with a side of 0.8 arcsec, covering the wavelength region 0.95$1.7 \mu \mathrm{m}$ at low spectral resolution. Additionally, this instrument will provide intermediate 


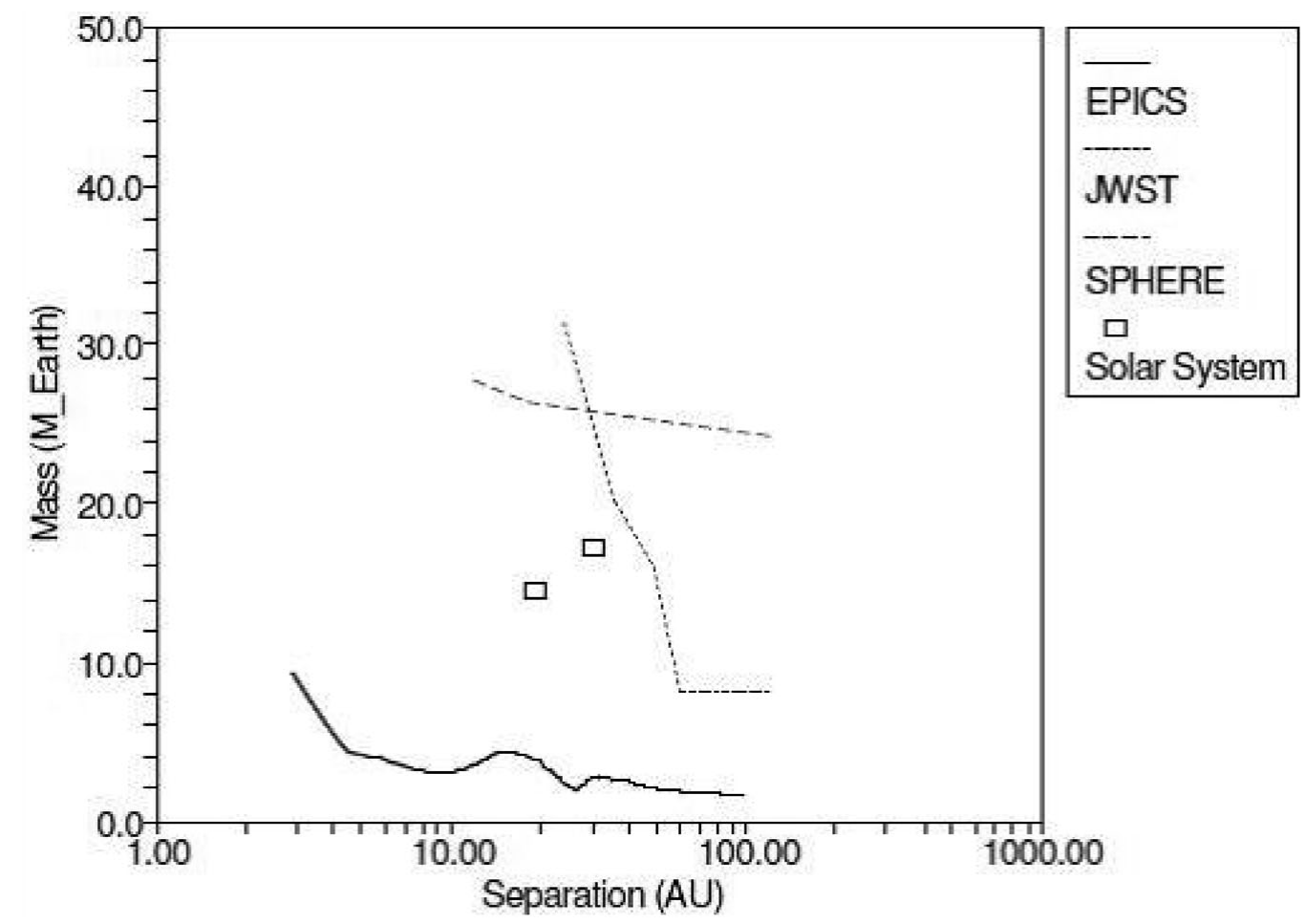

Figure 1. Limiting detectable mass of planets as a function of physical separation around a $10 \mathrm{Myr}$ old G2V star at a distance 120 pc. Observations potentially possible with EPICS and SPHERE are in the J-band; those with JWST are in the L band (data from Green et al. 2005). The location of the two outer giant planets of the Solar System (Uranus and Neptune) in this plane is shown for comparison.

$(R \sim 4000)$ and high $(R \sim 20,000)$ spectral resolution for follow up observations of bright planets. A polarization analyser (EPOL) will also be included, based on a concept similar to the ZIMPOL one under development for SPHERE. EPOL will yield high precision $\left(10^{-5}\right)$ differential polarimetry over the whole FoV, Nyquist sampled at diffraction limit, over the wavelength range $600-900 \mathrm{~nm}$.

\section{Science goals for EPICS}

EPICS will be a powerful instrument for detection and characterization of exosolar planets. Its design will be optimized in order to reach very significant goals in a number of areas. In this section we define the main goals of EPICS. This requires a quantitative estimate of the results we might expect from EPICS. At INAF-OAPD we developed a special software tool (MESS: Bonavita et al. 2010, in preparation) that allows making such quantitative assessments.

\subsection{Young self-luminous gas planets}

Detection of young self-luminous giant planets allows to determine the initial distribution with mass and separation, to be compared with models of formation and evolution. This is crucial, because these mechanisms are still far from being properly understood. The peak of the distribution of giant planets with separation is expected to lie slightly out of the so-called snow-line, where ices can survive, providing a wealth of material for the 
formation of large planetary cores: for solar type stars, the snow-line is expected to be at $\sim 3-5$ AU. Exploration of regions even further out (at $>10$ AU) provide information on the impact of neighbours on the dynamical evolution of the orbits of already formed planets. These regions are inaccessible with most methods. Direct detection may be very helpful because (i) a single image is enough for describing main characteristics of the whole system; (ii) repeated visits may allow determination or at least constraints on the main orbital parameters; and (iii) possibly coupled with indirect methods, planetary masses can be derived.

For this goal, best data are obtained for star forming regions and young associations, because very young planets are expected to be bright. These regions are typically at distances between 100 and 150 AU, although TW Hya is closer. Note that only a handful of stars in each of these regions are bright enough to be observable with EPICS.

Instruments on 8-10 m class telescope (SPHERE and GPI) should allow detection of a few tens giant planets around a few of these objects (see Figure 1), while JWST can observe farther stars and has a sensitivity to much smaller planet masses but only in the very outer regions of the systems. While this would be by itself of very high interest, the much higher sensitivity of EPICS should allow to observe much fainter planets, that is both less massive and/or older, and moreover to explore with high sensitivity much inner regions, close to the snowline. Figure 1 suggests in fact that Neptune-like planets should be detectable by EPICS down to a few (2-3) AU even if the limiting contrast is only $10^{-6}$. EPICS should then allow a complete census of the gaseous planets that form outside of the snowline.

The presence of disks, while providing very important information on their relation with the very young planets, may prevent planet detection. This may occur either because the disk is optically thick or because the disk is so luminous to overcome planet emission. Optically thick disks are observed around very young objects (age $\sim 1$ Myr or less) in the Orion nebula and elsewhere (see e.g. ODell \& Wen 1994). Observation of planets should then be easier around older objects, preferably seen pole-on (it is anyhow dubious that planets can form on such short timescale). Competition with planet emission is very critical for mid-IR instruments on 8-10 m or smaller class telescopes; it is less a problem with EPICS at E-ELT because observations are in the NIR, where disk thermal emission is negligible and the diffraction peak is more concentrated. Still, stellar light scattered by the disk may be a not negligible contribution to the local background, contributing to noise. For instance, in the case of AU Mic (a 12 Myr old M1V star), the near edge-on disk is $\sim 9 \mathrm{mag} / \operatorname{arcsec}^{2}$ fainter than the star (in the $\mathrm{J}, \mathrm{H}$ and $\mathrm{K}$ band) at $10 \mathrm{AU}$ from the star (Fitzgerald et al. 2007). Over the area subtended by a diffraction peak, the disk should then produce a flux equivalent to that of a planet with a contrast of $3 \times 10^{-9}$. This is close to the limiting contrast obtained by EPICS.

It should be noticed that young massive planets will be detected at quite high $\mathrm{S} / \mathrm{N}$ with EPICS. This means that they can be targets of follow-up observations with higher spectral resolution.

\subsection{Mature planets in the solar neighbourhood}

Observation of samples of giant planets in the solar neighbourhood is very important for various reasons: (i) Frequency and mass distribution of giant planets at old ages, once dynamic evolution have cleaned systems from planets in unstable orbits, can be compared with the results obtained in star forming regions and young associations. (ii) These systems may be studied in more detail, even in regions much closer to the central star with respect to the snowline, allowing exploring the habitable zone (HZ) and even inner regions. (iii) These observations are important forerunner for spatial missions for 

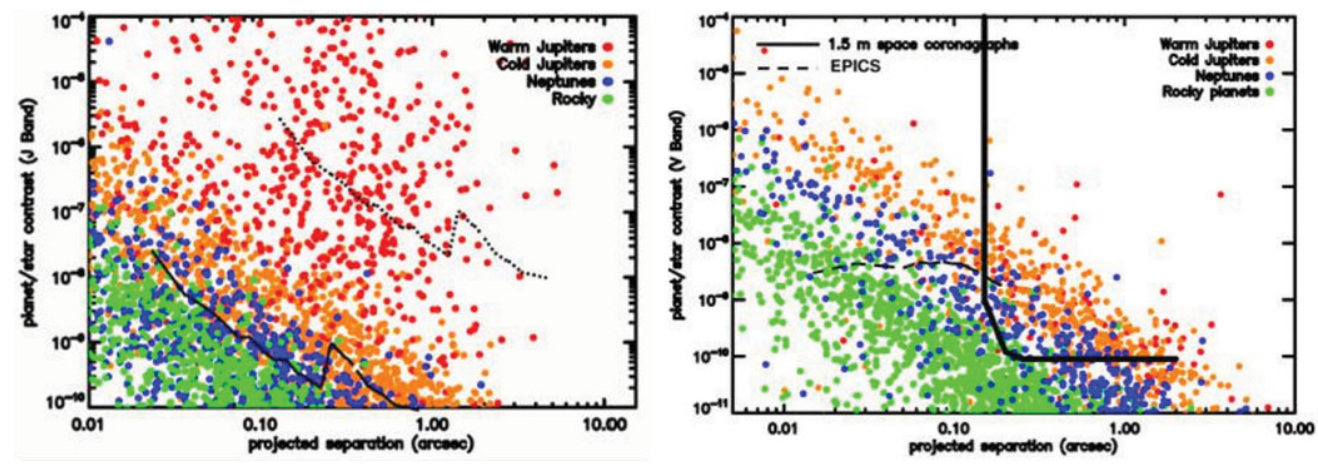

Figure 2. Left panel: Expected simulated planets in the plane contrast versus projected separation, compared with detection limits for EPICS IFS. For comparison, expected detection limits with GPI and SPHERE-ZIMPOL are also plotted (dashed lines). Right panel: the same for EPOL (dashed line). For comparison, expected detection limits with a $1.5 \mathrm{~m}$ space coronagraph are also plotted (solid line).
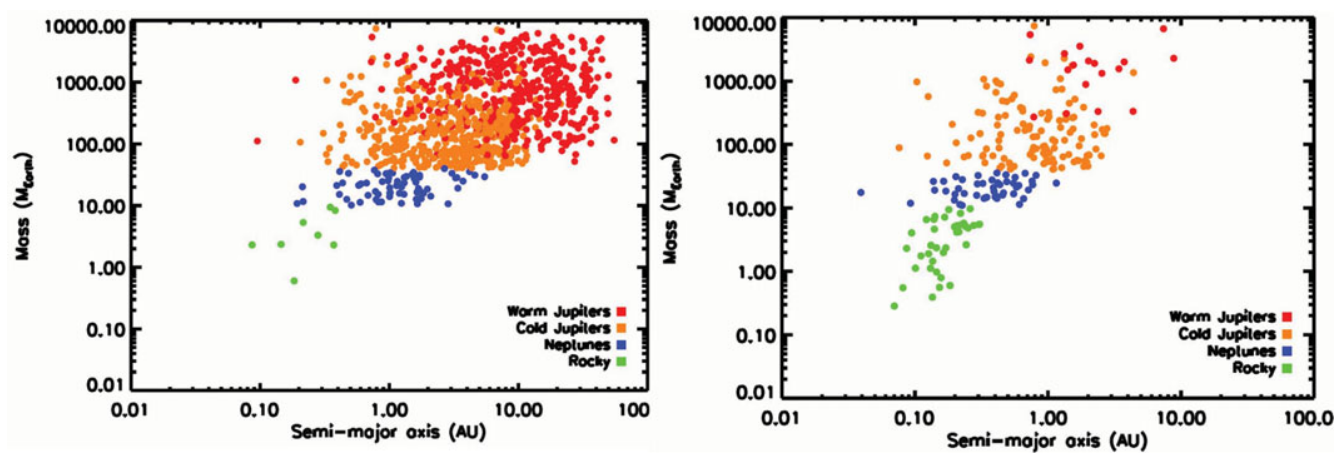

Figure 3. Simulations of planets detectable by the NIR IFS (left panel) and EPOL (right panel) of EPICS, plotted in the semi-major axis versus mass plane.

spectroscopy of Earth-like planets, clarifying which systems are most likely to host rocky planets in the habitable HZ.

Even more interesting would be detection of small mass planets (Neptunes and SuperEarths) that would allow to define the expected frequency of low-mass planets at various separations from the central star. Furthermore, even low resolution and S/N spectra of such objects would allow a first characterization of their atmosphere.

To examine the potentialities of EPICS for these scientific goals, we considered the properties of the planets expected to be detected on both a survey covering a sample of $\sim 600$ stars within $20 \mathrm{pc}$ from the Sun and brighter than $I<9$, and over a sample of 1200 young stars within 100 pc. For each star we randomly choose 5 planets (mass-semimajor axes) from the planetary population predicted by power laws extrapolated from RV surveys (Cumming et al. 2008). Luminosity of each planet is estimated taking into account both intrinsic luminosity and reflected light contribution. We then compared the expected contrasts with the curves for limiting detections: a planet is detected if it is above the detection threshold.

We distinguished different planet mass ranges: (i) all the objects with $M_{P}>40 M_{\text {Earth }}$ are considered as Jupiter-like planets; (ii) those with $10<M_{P}<40 M_{\text {Earth }}$ are the Neptune-like planets; (iii) and finally the ones with $M_{P}<10 M_{\text {Earth }}$ are considered as rocky planets. Figure 2.1 compares the expected distribution of planets in contrast 


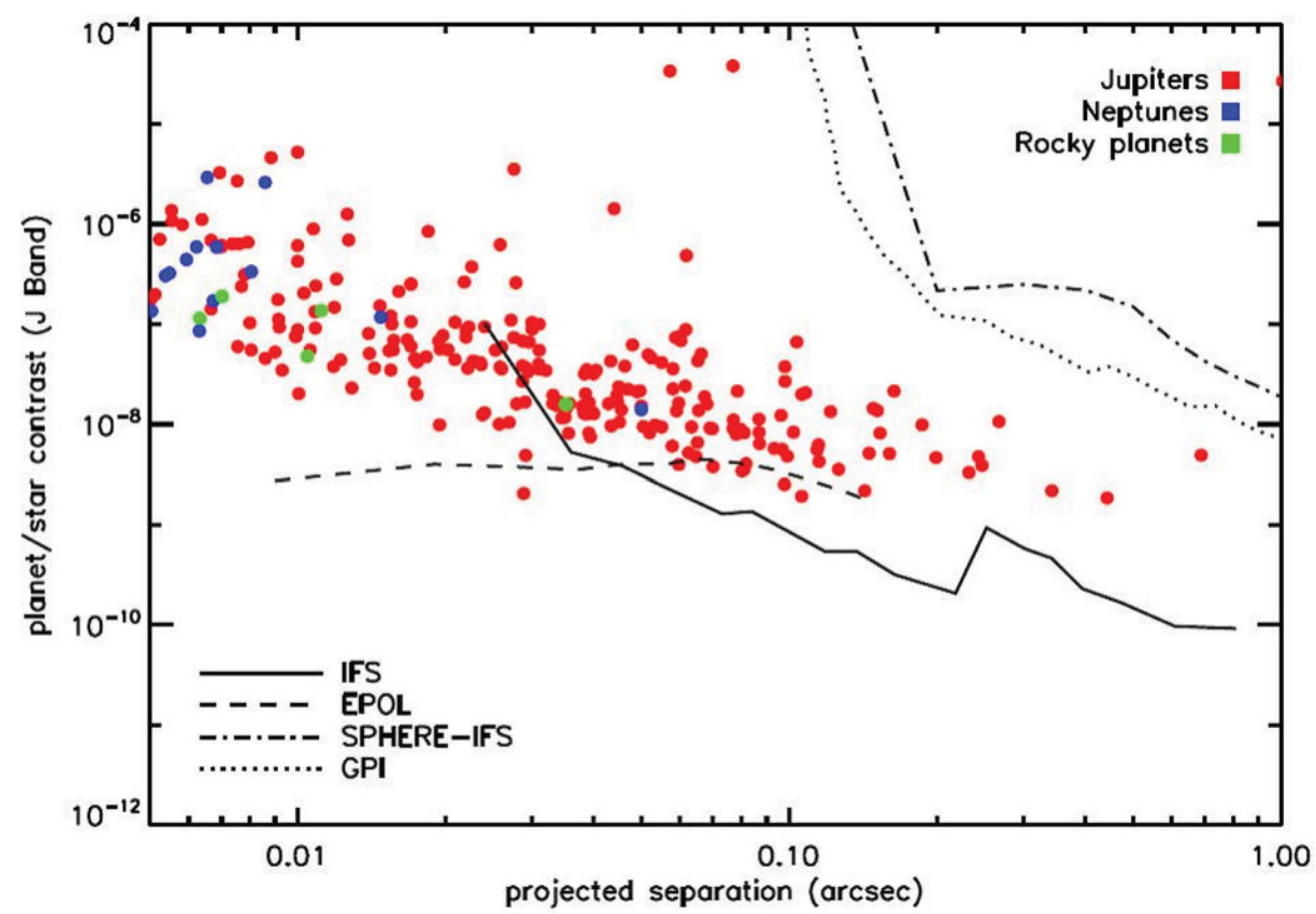

Figure 4. Exosolar planets already discovered with radial velocities in the separation-contrast plane in the J-band. Lines are detection limits with various high contrast instruments.

vs. projected separation plane, against limiting detection curves that might possibly be obtained using EPICS. Figure 2.1 shows the detected planets in the semi-major axis vs. mass plane. Essentially all Jupiter-like planets at projected separation beyond the EPICS IWA will be detected, making up a sample of several hundred objects. A similar survey will also be quite effective in detecting Neptune like planets, with several tens of them detected, in the range of projected separation from the IWA up to about 0.1 arcsec. On the other hand, in our simulations a few rocky planets are detected around very close and bright stars; for the IFS the success rate depends on random fluctuations as well as on the exact real performances of EPICS, while for the polarization analyser the IWA is very critical, and some tens of planets might be detected if this is as small as 0.01 arcsec. At the EPICS IWA (0.02 arcsec at best), most mature Super-Earths have a contrast below $10^{-7}$, and are below $10^{-8}$ at 0.1 arcsec, making them difficult objects at these small separations. We incidentally note that Gl581d is close to the expected detection limit for XAO at E-ELT. While considerable effort can be devoted to the observation of this single object, it is clear that EPICS performances should be put at their limit to allow such an observation.

We finally note that many detections are done even at rather high $\mathrm{S} / \mathrm{N}$. This implies that rather accurate spectral information could be gathered from these spectra.

\subsection{Planets discovered by $R V$, astrometry and transit searches}

In most cases masses for planets detected by EPICS cannot be determined independently of ages. Imaging of planets already detected by RV, transits and/or astrometry would represent a major breakthrough thanks to the availability of dynamical constraints (or even full orbit determination) on the planet masses and on the orbital elements. In most cases, stellar ages are or can be determined rather well exploiting suitable indicators 
(isochrones, magnetic activity and rotation, kinematics, etc.). Therefore, these objects will represent the ideal benchmarks for the calibration of models for sub-stellar objects. Spectroscopic and polarimetric observation of these planets (for which most important parameters are known) is crucial for testing models of their atmospheres. Given the distribution of separations for planets known from radial velocities, imaging of planets detected by RVs put constrains on both the IWA and on the contrast at very small separations. While planets at larger distances from the star are expected from RV surveys in the next years (stars that have clear trends of radial velocities being obvious candidates), it is clear that accessibility to planets at angular separations of $<0.05$ arcsec and with monochromatic contrasts of at least $10^{-8}$ is required in order to obtain images of a large sample of planets already discovered by radial velocities (see Figure 2.2). In addition, minimum semi-major axis decrease from $1.5 \mathrm{AU}$ for IWA=0.05 arcsec to $\sim 0.8 \mathrm{AU}$ for IWA $=0.02$ arcsec: this should allow obtaining spectra of objects in the HZ. On this respect, EPICS should represent a major step forward with respect to SPHERE, GPI, and JWST, that should be able to detect only very few if any of these planets. A few of these planets are several order of magnitude above the EPICS limiting contrast: very accurate characterization should be possible for such objects.

An interesting overlap region also exists with the planets that might be discovered by the PLATO mission. PLATO will allow to detect a large number of transiting planets around bright stars that could be studied in close detail. Planets down to about $10 M_{\text {Earth }}$ around M dwarfs with magnitude about $V=8.5-10$ (the bright end of PLATO) can be detected also with EPICS. The availability of planet spectra from EPICS and planet radii from PLATO will be extremely relevant for the physical study of the planets. For $\mathrm{G}$ and $\mathrm{F}$ stars (and $\mathrm{K}$ and $\mathrm{M}$ dwarfs as well) planets at separation larger than that accessible to PLATO can be detected, allowing to study the outer planetary system of PLATO targets.

\section{References}

Baraffe, I., Chabrier, G., \& Barman, T. S. 2003, A $\mathscr{E} A$, 402, 701

Beuzit, J.-L., Feldt, M., Doheln, K., et al. 2008, Proc. SPIE, 7014, 41

Cumming, A., Butler, R. P., Marcy, G. W., et al. 2008, PASP, 120, 531

Fitzgerald, M. P., Kalas, P. G., Duchêne, G., et al. 2007, ApJ, 670, 536

Green, J., Beichman, C., Basinger, S., et al. 2005, Proc. SPIE, 5905, 185

Marois, C., Macintosh, B., Soummer, R., et al. 2008, Proc. SPIE, 7015, 47

ODell, C. R. \& Wen, Z. 1994, ApJ, 436, 194 